\title{
Periodontal Experimental Research
}

\author{
Santosh Kumar Bangalore Balaram ${ }^{1,2}$ Arvind Babu Rajendra Santosh ${ }^{3} \quad$ Sushma Ravindra Galgali ${ }^{4}$
}

${ }_{1}^{1}$ Periodontist \& Implantologist, Private Practice, Kuwait

${ }^{2}$ Former Faculty Member, Department of Periodontics \& Oral Implantology at M.R Ambedkar Dental College \& Hospital, Bangalore, Karnataka, India

${ }^{3}$ School of Dentistry, Faculty of Medical Sciences, The University of the West Indies, Mona Campus, Kingston, Jamaica, West Indies

${ }^{4}$ Department of Periodontics, V.S Dental College \& Hospital, Bangalore, Karnataka, India

Eur Dent Res Biomater J 2020;1:64-71

\begin{abstract}
Address for correspondence Santosh Kumar Bangalore Balaram, BDS, MDS, FCOI, Private Practitioner, Kuwait City 35092, Kuwait (e-mail: drbbsantosh03@gmail.com).
\end{abstract}
Abstract
Keywords
- dental research
- periodontology
- experimental studies
- periodontitis
- therapy

Periodontal research in the last few decades has shown discoveries and inventions related to techniques and material science. In the first part of the review, we have discussed the problems and difficulties faced in epidemiological research. In the second part, an overview of the difficulties encountered in experimental periodontal research has been discussed.

\section{Introduction}

Periodontal experimental research has grown enormously over the decades, especially in relation to diagnosis and treatment of periodontal and peri-implant diseases. Researchers have faced numerous problems in executing experimental periodontal research due to various reasons such as nonavailability of suitable animal models, comparative differences between in vitro and in vivo research, inherent problems faced during histological research, and clinical research-related issues, mainly the ethical issues and sponsored research. In addition, there are other important advancements in the field of periodontology with respect to clinical microbiology, immunology, periodontal regeneration, stem cell therapy, and vaccine development. The aim of this paper is to discuss the various difficulties faced during experimental periodontal research.

\section{Problems Encountered with the Use of Laboratory Animals}

The most challenging aspect of studying periodontal disease is the "chronic nature" of the disease. Host responses to the periodontal microflora are complex, and research has shown that host-microbial interaction acts as a potential tool to limit as well as aggravate the disease. Furthermore, periodontitis on humans can only be studied in a retrospective approach due to the nonavailability of reliable clinical markers for studying ongoing connective tissue destruction including alveolar bone loss. Hence, animal models were considered in microbiological, immunological, and clinical studies related to periodontal diseases. Animal studies are effective complementary for in vitro experiments prior to testing new clinical treatments. Animal models should make a possible validation of hypotheses and prove the safety and efficacy of new regenerating approaches using biomaterials, growth factors or stem cells. ${ }^{1-3}$

\section{Murine Models}

Murine models are cheaper, easier to handle, and have genetically characterized strains. The murine calvarial model was developed by Brenden Boyce ${ }^{4}$ to study the effect of cytokines on bone resorption. This model does not contain a periodontium, and since the bacteria are actually delivered into soft tissue, their viability, colonization, invasion, or mechanism of infection cannot be studied.
DOI https://doi.org/ 10.1055/s-0040-1713945.
(C) 2020. European Dental Research and Biomaterials Journal.

This is an open access article published by Thieme under the terms of the Creative Commons Attribution-NonDerivative-NonCommercial-License, permitting copying and reproduction so long as the original work is given appropriate credit. Contents may not be used for commercial purposes, or adapted, remixed, transformed or built upon. (https://creativecommons.org/licenses/by-nc-nd/4.0/)

Thieme Medical and Scientific Publishers Pvt. Ltd., A-12, 2nd Floor, Sector 2, Noida-201301 UP, India 


\section{Murine Oral Gavages Model}

This model was developed by Pamela Becker. ${ }^{5}$ Mice are treated with antibiotics to deplete their microflora, followed by infecting the mice with microorganisms to induce bone loss. It is a nonsurgical model used to study host bacterial interactions and bone resorption. This model does not represent the chronic nature of the disease and is strain and gender dependent.

\section{Humanized Peripheral Blood Leukocytes (HuPBL)- Nonobese Diabetic (NOD) Mice}

This model was developed by Andy yen-Twig Teng. ${ }^{6}$ In this model, NOD mice are reconstituted with HuPBL engraftments from aggressive periodontitis subjects, followed by oral inoculation of Aggregatibacter actinomycetem comitans (A.a comitans), which leads to increased expression of the osteoprotegrin ligand (OPG L), a key mediator of osteoclastogenesis and osteoclast activation. ${ }^{2}$

\section{Rat Models}

Rat models have gained popularity because of large oral cavity and teeth, allowing the taking of more sample specimen material and quantifying bone changes. Rovins et al said rat ligature models have been used for many decades for experimental periodontitis. ${ }^{7}$ The silk or cotton ligature is placed around the tooth, facilitating plaque accumulation. In germ-free rats, it is not the ligature but the increasing Gram-negative microorganisms that enhance osetoclastogenesis and bone resorption. The mechanism of bone formation and resorption, coupling and host bacterial interactions can be studied in this model but isolation of bone is difficult, and the ability to effectively infest and maintain the infection during an interval to create bone resorption remains a challenge. In oral infection model, rats are orally infected on repeated occasions to establish colonization. It allows studying immune cell responses and bone resorption. But isolation of immune components is not possible, and there is limited ability to dissect the individual immune cell contributions to periodontal bone resorption compared with the murine model. ${ }^{1,2}$

\section{Immune Cell Models}

Immune cell models were developed by Martin Taubman. In the T cell model, bone resorption can be quantified after 10 days of cell transfer. The rats are subjected to euthanasia in order to further study the parameters of bone resorption. In case of the B cell model, normal inbred rats are injected intraperitoneally with antigen (whole A.a bacteria). Neither of the models represent a chronic periodontal infection. Bone resorption is dependent on the concentration of transferred $T$ cells or B cells used in the study. ${ }^{8}$

\section{Lipopolysaccharide (LPS)-induced Alveolar Bone Resorption Model}

This model was developed by Keith Kirkwood. It is an established model of aggressive inflammatory alveolar bone loss in rats using LPS derived from periodontal pathogens like A. a comitans. The LPS is delivered orally three times a week, and bone loss is observed within 8 weeks and quantitatively measured. This is not an infectious chronic model but an inflammation-induced bone loss model, as only LPS-induced bone loss can be tested., ${ }^{1,2}$

In hamsters, the inflammatory response is not only very limited but also very different from that observed in humans. The mechanisms of alveolar bone resorption in hamsters with diet-dependent periodontal lesions are quite similar to those observed in rats infected with Gram-positive bacteria. The rats and hamsters have different dental formulas than humans. The continuously erupting and drifting of teeth throughout the life of the rodent makes it a questionable periodontal model. The pathogenesis of periodontal disease follows a different pattern, and different immune responses are involved. They are susceptible to caries when inoculated with a cariogenic oral flora and fed with a high-sugar diet. This diet will also result in formation of calculus. Therefore, rats and hamsters could be suitable for caries and calculus research. Furthermore, gnotobiotic rats and conventional hamsters have been used to investigate the ability of microorganisms to cause destructive periodontal disease. ${ }^{1,3}$

\section{Dog Model}

Many experimental studies on gingival and periodontal diseases have been conducted in dogs. The beagle is one of the most commonly used dogs, due to its size and extreme cooperative temperament. Globally, all periodontal tissues and the size of the teeth are quite similar to those observed in humans. However, some major differences exist between dogs and humans due to the lack of lateral movements, no occlusal contacts for all the premolars, and presence of open contacts between teeth. The frequent lack of gingival sulci and crevicular fluid, a different composition of periodontal plaque and calculus are other important differences between dogs and humans. ${ }^{9}$

The prevalence and severity of gingivitis and periodontitis in dogs increase with age but vary markedly between the different breeds. Some dogs are susceptible to periodontal disease and others are more resistant. The differences can be explained more by the nature of the infection or genetics than by diet. In assessing natural periodontal diseases, as the extent and localizations of the periodontal lesions are not homogeneous, it may be considered to be a limitation of the model. Dog models are expensive with limited availability of bony defects and experience faster bone formation.,10

\section{Nonhuman Primate Model}

They represent heterogeneity of genetic background comparable to humans. The ligature-induced disease model in primates are imperfect relative to human disease, but clearly represent the most accurate model of complex microbial film, as it localizes host inflammatory/immune responses and tissue destruction commensurate to that of periodontitis. Also, this model enables studies of vaccine approaches to control the commensal bacteria and help in better understanding of the disease process. However, it entails high-cost, specialized facilities, need for euthanasia, and uniquely trained personnel to handle these ferocious animals. Wild captured 
monkeys can be disease carriers (zoonotic infections), and prone to systemic infections and diseases. They pose difficulties in controlling postsurgical infections and trauma. Finally, immunological studies that require synergistic host-cell interactions cannot be done in primates. ${ }^{1,3}$

\section{Limitations of Animal Models}

a) Animal research and its value to human experience remain controversial. Regardless of how much data can be presented, it is impossible to expect different species to respond identically or even similarly to the same challenge except within very narrow limits.

b) Research animals are very expensive to acquire and care for and are only used because no alternatives currently exist.

c) Genetic background of many of the animals has not been established.

d) Animals used in research exhibit heterogeneity in age, body weight as well as oral and general health conditions.

e) Features of periodontal diseases in humans and animals vary greatly, depending upon which form of the disease is present and the stage of the development.

\section{In Vitro Versus In Vivo Research}

In humans, there exists a complex system that has been validated for a true causal study of infection, host response, and disease progression, which can be accomplished over a reasonable time frame. Hence, in an in vivo study, factors such as host defense, host-bacterial interactions, and intercellular heterogeneity may be present. But in in vitro studies, there is a void of these factors and therefore, both experimentally and statistically, the inference may vary. In vivo models also have their own limitations such as a) inability to design a model that harbors the exact type of defect seen in human disease, b) further, many models use an acute defect (such as critical size defect model) to determine whether a factor does elicit a response, and may not actually reflect the response for chronic situations, such as those found in periodontitis patients, c) moreover, there are distinct genetic, anatomic, biochemical, immune, and microbial differences between the species. In attempts to overcome these drawbacks, genetically engineered animals and cells in vitro and in vivo are being used. ${ }^{11}$

\section{Problems in Histological Research}

A biopsy is the removal of part, or all, of a lesion to enable histopathological examination and definitive diagnosis. The issues related to histological research are associated with artifacts in mucosal biopsies. ${ }^{12}$ The troubleshooting problems in the histological research can be categorized as preoperative, operative, postoperative, and procedures in the histological technique issues. It is noteworthy to mention that the structures that are viewed under microscopic examination of biopsy specimens are not always related to the normal histology or pathology, but also due to the defects related to the surgical procedures, fixation, transportation of specimen, and processing, sectioning and staining of the tissue specimens..$^{13}$ Thus, careful handling of the soft tissues is imperative to ensure the pathologist receives a representative sample of the lesion and make an accurate diagnosis. ${ }^{14}$ The application of biopsy in the management of oral lesions includes a sequence of steps: adequate data collection, competent diagnostic skills, proper surgical management, evaluation and interpretation of the pathologist's report, and comprehensive patient follow-up. For the purpose of convenience, we are discussing the problems in histological research under three headings: 1 ) problems in simple histological research, 2) problems in routine histological research, and 3) problems in advanced histological research.

\section{Problems in Simple Histological Research}

Cytology is a key component in the diagnosis and screening of malignant conditions. This procedure assesses single or clusters of cells, which is smeared from the lesion. Cytological staining method is a simple histological research procedure. The most common type of artifact that is encountered in cytological preparation is due to inappropriate handling of the sample before the fixation procedure, or due to contaminants in the staining material. The inappropriate contact between slide holder and a cytological smear, which is wet, may result in cardboard or packing artifact. ${ }^{15}$

\section{Problems in Routine Histological Research}

Routine histological research includes hematoxylin and eosin ( $H$ and $E$ ) staining, which remains the gold standard method in histoinvestigation. Various artifacts that are included in the $\mathrm{H}$ and $\mathrm{E}$ staining are categorized into preoperative, operative, postoperative, and artifacts related to the histological techniques. The description of this section is divided into the following two headings: 1) Artifacts related to preoperative, operative and operative periods of biopsy procedure and 2) artifacts related to histological techniques.

\section{Artifacts Related to Preoperative, Operative and Postoperative Period of Biopsy Procedure}

The tissue alterations may be encountered due to certain preoperative and operative issues. These include the following: 1) the surface preparation of the biopsy site with surgical marking inks such as iodine and 2) the local anesthetic solution, which is infiltrated around the biopsy site, may cause the swelling of the cells and tissues in the lesional area. The improper tissue handling during surgical biopsy procedure may cause tissue damage, needle marks, cautery, and crushing of the tissue. The exact relationship of the epithelium and connective tissue may be lost if the surgical incision is too shallow and if the injection of anesthetic solution can produce hemorrhage with extravasation. Anesthetic solution deposition may often show vacuolization changes between epithelium and stroma, which gives an artifact observation of the subepithelial split formation. Curling of the biopsied tissues are more commonly observed in keratotic/hyperkeratinized lesions. ${ }^{16}$ Sometimes, curling of the biopsied tissue may be noted in the small-sized tissue 
samples. Foreign bodies may get entrapped during preoperative/operative procedures, which includes iodine, glove material, and cotton. The postoperative artifacts are mostly tissue shrinking, bacterial contamination, and tissue death. The shrinkage of tissue may be most commonly noted in the samples stored in saline solution. The bacterial contamination of the biopsied tissue is due to inadequate removal of blood in the tissue, resulting in ineffective tissue for staining procedures. ${ }^{17}$ The tissue death may be due to the improper removal of blood from the biopsied tissue or expired fixative solution.

\section{Artifacts Related to the Histological Techniques}

The biopsy procedure is followed by a sequence of histological technique such as fixation, tissue processing, embedding, microtomy, staining, and mounting. The artifacts may incur during any of the stated steps of histological technique.

The fixation is a procedure to seize autolysis and putrefaction, and stabilize the proteins present in the biopsied tissue. Thus, fixation facilitates in a good preservation of cellular architecture by retaining the proteins present in the cell or tissue. In most instances, the tissues are fixed in $10 \%$ formalin solution for 2 to 10 hours. The volume of the solution should be maintained at 20 times higher than the biopsied tissue volume. ${ }^{18}$ The delayed fixation or delayed transportation of biopsied tissue into the fixative solution will lead to cellular shrinkage, loss of nuclear material, and cytoplasmic clustering. On the contrary, prolonged fixation leads to secondary tissue shrinkage and hardening. Freezing during transportation may direct to cytoplasmic condensation. ${ }^{19}$

Dehydration and clearing are the steps involved in the tissue process. The process of removal of hydrous or aqueous content of the tissue is termed as dehydration. Dehydration is usually achieved by using alcohol solution. During the dehydration process, the aqueous content is replaced by dehydrating agent (alcohol). ${ }^{20}$ The tissues immersed in dehydrating agent usually demonstrate shrinkage due to removal of aqueous content, and this is termed as shrinkage artifact. Dehydrating agents such as acetone, if used over a prolonged period of time, may increase the brittleness of the tissue and subsequently cause sectioning artifacts. The process of removal of a dehydrating agent from the tissue, which is followed by replacement of fluid that is miscible with dehydrating agent and embedding medium, is termed as clearing. Prolonged processing of tissue in clearing agent may increase the tissue brittleness, predisposing to disintegration or crystallization of tissue during sectioning or microtomy procedure. ${ }^{21}$

Embedding is a step where the processed biopsied tissue is placed in the paraffin wax while preparing tissue block to conduct sectioning or microtomy procedure. Inappropriate orientation of the tissue during the embedding procedure may lead to missing important and significant elements of biopsied tissue for microscopic observation. ${ }^{21,22}$ The tissues should be immediately transferred from paraffin wax solution to the embedding procedure; a prolonged wait period in paraffin wax may cause tissue hardening and increase the brittleness. Inadequately dehydrated biopsied tissues are prone to tissue hardening during the embedding step, which may result in tearing artifact during the sectioning procedure. ${ }^{22}$

Microtomy is the process of tissue sectioning, which enables microscopic observation upon staining of the biopsied tissue. Wrinkling, curling, nicks in tissue, and alternate thick and thin sections are the procedural artifacts in microtomy. ${ }^{23}$ Inadequately embedded tissues usually provide the challenges during sectioning procedures and results in thick or thin section. The knife used in the microtomy procedure may also cause artifacts such as nicks in tissue and thick or thin sections. ${ }^{24}$

Staining is the process where the cellular structures take up the stain, enabling specific microscopic appearance. The usual staining artifacts are due to excessive staining, outdated stain material, and decomposed dyes or contaminated stain. Blotching in the stained tissue sections is observed in the areas of inadequate removal of paraffin wax. ${ }^{25}$

Mounting is a process where the stained tissue sections are protected by a cover glass with mounting media. The mounting media are usually resinous content. Bubbling is the common artifact in the mounting procedure. Bubbling is resultant of air gaps between the cover glass and tissue section. $^{26}$

\section{Problems in Advanced Histological Research}

Immunohistochemistry or immunocytochemistry is the process of detecting antigens in cells of a tissue or cytological section by antibody-antigen interaction. Immunohistochemistry has a great application in diagnosis of malignant lesions. Artifacts in immunohistochemistry techniques are listed as edge and trapping artifacts, desquamation artifacts, bubble artifacts, drying artifacts, artifacts of poor fixation, precipitate DAB artifacts, biotin artifacts, inadequate background staining, limited background and general background. The most common artifacts in immunohistochemistry technique are due to inadequate background staining, and limited background and general background staining will be discussed below.

Little or no staining of controls and/or specimen tissue, except for counter stain, is termed as inadequate background staining. The inadequate background staining results due to any one of the following reasons: omission of primary antibody or labeled reagent, reagent used in incorrect order, diluted or concentrated reagents, inappropriate incubation time and temperature, lack or stabilizing or carrier protein, detergent in diluents, defective or outdated primary antibody or secondary antibody, dissociation of primary antibody due to excessive washing, use of alcohol based counter stain, or incorrect substrate-chromogen mixture. ${ }^{27}$

The areas of inconsistent staining of control and/or specimen tissue are termed as limited background. The limited background may be result due to one of the following reasons: protein trapped beneath the tissue during the mounting process, pooling of immunohistochemistry reagents beneath the tissue section, partial detachment of the tissue from the slide, undissolved chromogen molecule, incomplete removal of embedding medium, bacterial or yeast contamination 
from mounting water bath, or partial drying of tissue prior to fixation. ${ }^{27,28}$

Background staining may be seen in epithelial or connective tissue elements of control tissue and/or specimen. The false positive brown staining in the background is due to one or more of the following reasons: excessive incubation with substrate-chromogen reagent, incorrect preparation of substrate chromogen reagent, concentrated secondary antibody, inadequately rinsed slides, insufficient saline, detergent in buffer, inappropriate blocking serum, nonspecific binding of secondary antibody with tissue specimen, hydrophobic and electrostatic interactions, endogenous peroxidase, endogenous biotin, or necrotic tissue. ${ }^{27,29}$

\section{Difficulties Encountered in Clinical Research: Problems Faced in the Previous Classification "a Disease or Syndrome"?}

Although numerous classifications have been proposed over the years, it can be argued that none of them serve their purpose, due to the overlapping criteria used to define the cases and determine the extent and severity of periodontitis. Hence, diagnostic tests designed to distinguish different types of periodontitis have not been developed due to the overlapping issue.

Development and validation of tests would be extremely helpful in the treatment and prevention of periodontal infection. ${ }^{30}$ At present, we are handicapped in making precise diagnosis and prognosis, as there are no reliable markers of disease activity and no reliable criteria for identification of the risk. Currently, we are still looking for high-precision tools to diagnose and classify the disease presented by a patient on an etiological basis.

\section{Diagnostic Tests}

Diagnostic tests that dichotomize disease state into yes or no category and the test result into positive or negative do not represent clinical reality. Partial mouth recordings or charting from a selection of teeth/sites might overestimate or underestimate disease compared with full mouth registrations. Utilizing only the site as the unit of observation exaggerates the statistical power, suggesting wrongful effect of therapy and overrepresentation of the perception of disease. Conventional periodontal diagnostic methods are incapable of providing any information on the cause of the condition, on the patient's susceptibility, disease progression, or whether the response to therapy will be positive or negative. The drawbacks of conventional diagnostic methods include clinical or radiological measurements of attachment loss not being precisely accurate, and if not performed very carefully, they might be misleading. All clinical diagnostic techniques provide only retrospective information about past diseases and are unable to diagnose disease activity. Therefore, advanced diagnostic methods have been developed, which in spite of being superior have inherent problems. ${ }^{31,32}$

\section{Analysis of Diagnostic Markers for Periodontitis}

Use of biological diagnostic marker should have high-specificity and sensitivity, and one should be able to use it easily at the chair side or as a home use device or test. No factor in gingival crevicular fluid (GCF) or in saliva has been identified, which can distinguish between aggressive or chronic periodontitis; although, a multitude of these markers have been studied in both types of disease.

\section{Problems in Imaging Methods in Periodontology}

Radiography, even when used appropriately and optimally, is far from perfect. Due to lack of accuracy, as with any diagnostic test, there is a chance that false-positive or false-negative results will occur. The limitations are with standardization, projection geometry, scattered radiation, and unfavorable cost-benefit ratio in digital imaging.

\section{Problems with the Measurement of Periodontitis or End Point in Clinical Trials}

The lack of reproducibility in measurements used in clinical trials is probably the one of the factors that can explain disagreement between clinical studies. Even if there was an agreement on the methodology of measurement of periodontitis, there is no consensus on clinical significance. A fundamental issue in the clinical trial designs are the distortion between true and surrogate end points.

True end points are tangible outcomes that directly measure the patient's perception of the disease symptoms or the treatment outcomes. The true end points are sometimes referred to as clinically relevant endpoints/clinically meaningful end points/terminal endpoints, or ultimate endpoints. True end points are usually subjective such as pain, bleeding on brushing, and oral health-related quality of life measures (for example, reduction of halitosis, teeth hypersensitivity, tooth mobility after treatment, etc.).

Surrogate end points (intermediate endpoints/biological markers/biomarkers) are physiological or biochemical markers that are easier to measure than true end points. These end points are usually objective and intangible to the patient's mind. Typical surrogate end points in periodontal research include anatomic measures (e.g., probing depth), clinical attachment level, measures of inflammation, microbiological measures, and immunologic measures.

The use of surrogate end points can lead to both false-positive and false-negative conclusions. Pivotal trials based on true end points may be of a long duration and require the recruitment of thousands of patients. True end point-based trials are suitable for evaluating short-term treatment goals such as aesthetic surgical procedures, splinting of mobile teeth and medications for painful periodontal abscesses. Subjective true end points are rarely used in clinical trials because of the notion that objective surrogates are superior to true end points. Nevertheless, the goal of therapy should be to provide tangible patient benefit, not tangible clinician benefit. $^{33}$ 


\section{Clinical versus Statistical Significance in Periodontal Research}

Currently, there is a great reliance on using statistical significance testing or hypothesis testing, to detect a statistically significant difference between therapies, which is used to infer a clinically meaningful result. Statistically significant results of the studies may be clinically insignificant to the clinician and the patient. Hence, there is a need to define a set of criteria that would reflect the important therapeutic changes of clinical relevance to the practitioner.

\section{Statistical Significance}

The term "statistical significance," suggests that the associations between tested variables did not occur by chance. The term "statistically significant at $\alpha$ level of.05" means that the null hypothesis (that there is no relationship regarding specific variables between test and control groups) was rejected and the chances of the association occurring by chance was small (probability is $5 \%$ or less, or the odds are 1out of 20 ). The major factors that affect the statistical significance are as follows

A. Effect size (mathematical determinant)-It is a statistical concept that measures the strength of the relationship between two variables on a numeric scale.

B. Degree of variation in the outcome measure between individuals,

C. Number of subjects used in the study.

D. Study design factors such as eligibility criteria, method of randomization, and control of confounders in observational studies.

\section{Clinical Significance}

A major dilemma in data interpretation from periodontal clinical trials is the insufficient standardization of quantifying clinical significance. This has resulted in using arbitrary statistical standards to define the merits of therapeutic techniques. As periodontal diseases are heterogenous in nature, and different treatment modalities may have different clinical outcomes, there is a need to define clinical significance for the response to therapies.

Clinical significance depends on the following factors:

A. Size of treatment effect: The larger the likelihood of obtaining an expected benefit of treatment (relative to a control treatment), the more clinically significant is the treatment.

B. Cost.

C. Side effects.

D. Time needed for therapy.

E. Ease of implementation.

F. Duration of results.

G. Consumer acceptability.

To arrive at a conclusion that a result is clinically significant, the finding must be clinically meaningful and statistically significant. Clinical trials are clinically significant when they address the patient's oral health-related quality of life parameters such as retention of their teeth, comfort, good function of their teeth, lack of side effects, and resolution of their problems. ${ }^{34,35}$

\section{Ethical Issues in Sponsored Clinical Research}

The relationship between industry and academia has created a variety of situations which have the potential of leading toward ethical compromises.

Three categories of behavior of those dealing with ethical issues in science are as follows:

A. Scientific misconduct is defined to include activities such as the fabrication or falsification of data and/or plagiarism.

B. Conflict of interest is defined as "conflict between the private interests and the official responsibilities of a person in a position of trust" (Council on Scientific Affairs and Council on Ethical and Judicial Affairs, 1990). ${ }^{36}$

Conflicts of interest occur at different levels such as the following:

1. Pharmaceutical industry levels such as decisions to invest to develop new products, especially vaccines and drugs, and market these products.

2. Investigators' level-related conflicts such as financial gains to participate in pharma-sponsored trials, expected academic career boost attained with the publication of the results of the trials, and personal interests such as financial support for trips to international conferences. ${ }^{4}$

3. Universities or research institutes having conflicts of interest, as the sponsored projects may help increase their budgets, both directly (taxes) and indirectly (e.g., improvement of physical infrastructure of laboratories or outpatient clinics). ${ }^{37}$

\section{Inappropriate behavior.}

In defining the relationship between industry and academia, it is the obligation of the investigator and the sponsor to conduct studies according to the highest scientific standards to help ensure the validity of the results.

In the context of an industrial/academic relationship, the following situations may be considered as inappropriate:

$>$ The inability to maintain the confidentiality of proprietary information.

$>$ The inability to enroll subject populations in the timely manner in it which had been promised.

$>$ The arbitrary and unilateral alteration of a study protocol.

$>$ Other actions which might breach the trust implicit in the establishment of a relationship between a company and an investigator or institution. ${ }^{36,38-40}$

\section{Difficulties Encountered in Advanced Research (Issues Related to Therapy)}

\section{Stem Cells}

The inherent properties of stem cells including their ability to form different tissue types and self-renew make their presence within the healing periodontal defect desirable to facilitate periodontal regeneration. ${ }^{41,42}$ 


\section{Problems Associated with the Use of Stem Cells}

1. Moral and ethical issues in embryonic stem cell research, including therapeutic and reproductive cloning.

2. Stem cells are not available in large quantities and grow slowly. So, it will be difficult to create sufficient tissue, and isolate and purify them.

3. There may not be adequate time to cultivate enough cells for treatment.

4. Side effects following stem cell transplantation like growth of tumors through abnormal cell behaviors.

5. Problem of rejection. ${ }^{43}$

\section{Technical Difficulties in Gene Therapy}

a. Gene delivery: Successful gene delivery is unpredictable, even in single-gene disorders. For example, although the genetic basis of cystic fibrosis is well-established, the presence of mucus in the lungs makes it physically difficult to deliver genes to the target lung cells. Delivery of genes for cancer therapy may also be complicated by the disease at several sites.

b. Durability and integration: Few gene therapy approaches aim at long-term effects. Two possible ways of achieving this are to either use multiple rounds of gene therapy or integrate the therapeutic genes, so that they remain active for some time.Gene therapy has not been applied with success in periodontics, primarily because of problems with viral vectors like patient toxicity, immune and inflammatory responses, gene control, and targeting tissues. Also, limitations of sufficient quantity of the engineered gene that can be delivered, extreme cost, and ethical restrictions pose problems. ${ }^{44,45}$

\section{Issues Related to Periodontal Vaccines}

The following are the challenges faced and the strategies that could be considered in the periodontal vaccine research:

1. As periodontal diseases are multifactorial and polymicrobial in nature, a vaccine targeting multiple periodontal pathogens need to be developed. Currently, the majority of studies that can be linked to vaccine trials have been focused on P. gingivalis. ${ }^{46}$

2. The efficacy of the vaccine in every individual may not be similar, owing to the variations in the serotypes or genotypes of the organisms.

3. The results from vaccine studies in animal models cannot be directly generalized to humans, due to the qualitative differences seen with respect to the oral microbial ecosystem, histological components of the periodontal lesions, and nature of immune responses. Currently, the only matched animal models for periodontal vaccine research are nonhuman primates. ${ }^{47}$

4. Although research has shown significant levels of shortterm protection, a momentous challenge exists in maintaining immune memory to prevent reinfection. ${ }^{48}$

5. The functional differences between the antibodies produced by the infected host and that produced after immunization need to be considered during vaccine development, as the former is usually present but is ineffective in resolving the disease. Hence, a vaccine that can generate functionally viable antibodies should be one of the most desirable features of periodontal vaccine.

6. Toxic reactions against vaccines based on the use of killed bacteria are a concern. This could be managed using subunit vaccines that can induce low-levels of immunogenicity. But subunit vaccine preparations are often problematic due to contamination with other virulence factors. ${ }^{48,49}$

7. A common vaccine strategy for dental caries and periodontal diseases could be developed using composition of different antigens from different bacteria like triple combination vaccine, such as classical measles, mumps, and rubella virus vaccine combination, against three different diseases. ${ }^{46,47}$

\section{Conclusion}

Research should endeavor toward clinical utility; improvement in techniques, concepts and analysis may be introduced to sharpen our research abilities. To achieve this, a multilevel approach involving cell biologists, matrix biologists, pharmacologists, biomaterial scientists, genetic engineers, and nano technologists will be required to address the problems faced in periodontal research. Periodontists need to understand the strengths and weakness of research and then try to implement an evidence-based approach for the benefit of the patient. This is the best assurance that periodontal research can utilize to reach its greatest potential.

\section{Conflict of Interest}

None declared.

\section{References}

1 Weinberg MA, Bral M. Laboratory animal models in periodontology. J Clin Periodontol 1999;26(6):335-340

2 Public Health Service Policy on Humane Care and Use of Laboratory Animals. National Institutes of Health. Office of Laboratory Animal Welfare. U.S. Department of Health and Human Services. NIH Publication No. 15-8013

3 Struillou X, Boutigny H, Soueidan A, Layrolle P. Experimental animal models in periodontology: a review. Open Dent J 2010; 4:37-47

4 Boyce BF, Aufdemorte TB, Garrett IR, Yates AJ, Mundy GR. Effects of interleukin-1 on bone turnover in normal mice. Endocrinology 1989;125(3):1142-1150

5 Baker PJ, Dixon M, Evans RT, Roopenian DC. Heterogeneity of Porphyromonas gingivalis strains in the induction of alveolar bone loss in mice. Oral Microbiol Immunol 2000;15(1):27-32

6 Teng YT, Nguyen H, Hassanloo A, Ellen RP, Hozumi N, Gorczynski RM. Periodontal immune responses of human lymphocytes in Actinobacillus actinomycetemcomitans-inoculated NOD/SCID mice engrafted with peripheral blood leukocytes of periodontitis patients. J Periodontal Res 1999;34(1):54-61

7 Rovin S, Costich ER, Gordon HA. The influence of bacteria and irritation in the initiation of periodontal disease in germfree and conventional rats. J Periodontal Res 1966;1(3):193-204 
8 Kawai T, Eisen-Lev R, Seki M, Eastcott JW, Wilson ME, Taubman MA. Requirement of B7 costimulation for Th1-mediated inflammatory bone resorption in experimental periodontal disease. J Immunol 2000;164(4):2102-2109

9 Sorensen WP, Löe H, Ramfjord SP. Periodontal disease in the beagle dog. A cross sectional clinical study. J Periodontal Res 1980;15(4):380-389

10 Giannobile WV, Finkelman RD, Lynch SE. Comparison of canine and non-human primate animal models for periodontal regenerative therapy: results following a single administration of PDGF/IGF-I. J Periodontol 1994;65(12):1158-1168

11 Saygin NE, Giannobile WV, Sommerman MJ. Molecular and cell biology of cementum. Periodontol 2000;24:73-98

12 Moule I, Parsons PA, Irvine GH. Avoiding artefacts in oral biopsies: the punch biopsy versus the incisional biopsy. $\mathrm{Br} \mathrm{J}$ Oral Maxillofac Surg 1995;33(4):244-247

13 McInnes E. Artefacts in histopathology. Comp Clin Pathol 2005;13:100-108

14 Margarone JE, Natiella JR, Vaughan CD. Artifacts in oral biopsy specimens. J Oral Maxillofac Surg 1985;43(3):163-172

15 Woods AE, Ellis RC, Laboratory Histopathology: A complete reference. Churchill Livingston Publishers: Edin Burgh. 1994; 456-472

16 Ficarra G, McClintock B, Hansen LS. Artefacts created during oral biopsy procedures. J Craniomaxillofac Surg 1987;15(1): 34-37

17 L R Eversole. Laser Artifacts and Diagnostic Biopsy.Oral Surg Oral Med Oral Pathol Oral Radiol Endod 1997;83(6):639-40. Doi: 10.1016/s1079-2104(97)90308-5

18 Yellowitz J, Horowitz AM, Goodman HS, Canto MT, Farooq NS. Knowledge, opinions and practices of general dentists regarding oral cancer: a pilot survey. J Am Dent Assoc 1998;129(5):579-583

19 Kumar K, Shetty DC, Dua M. Biopsy and tissue processing artefacts in oral mucosal tissues. Int J Head Neck Surg 2012;3(2):92-98

20 Carleton HM, Drury RAB, Wallington EA, Carleton's Text book of Histological Techniques. 5th ed. The University of Michigan: Oxford University Press; 1967

21 Culling CFA, Allison RT, Barr WT, Cellular Pathology Technique. 4th ed London, Boston: Butterworths; 1985

22 Krishnanand PS, Kamath VV, Nagaraja A, Badni M. Artefacts in oral mucosal biopsies. J Orofac Sci 2010;2(1):57-62

23 Lolli R, Venezia A, Bellardini M, De Nisi S, Demuro G. [Technical artifacts in biopsy of the oral cavity. I. Clinical and histopathologic aspects]. Minerva Stomatol 1989;38(1):37-45

24 Sabater-Recolons M, Vinals-Iglesias H. Las biopsias en medicina oral. Rev Eur Odontoestomatol 1997;3:175-182

25 Saunders WH, Wakely P, Atlas of Head and Neck Pathology. Philadelphia, USA: Ohio State University; 1998

26 Rolls OG, Farmer JN, Hall BJ, Artifacts in Histological and Cytological Preparation. Scient ia Leica Microsystems Education Series; 2008

27 Ramos-Vara JA. Technical aspects of immunohistochemistry. Vet Pathol 2005;42(4):405-426
28 Atwood K, Trouble Shooting in Immunohistochemical Staining Methods. 4th ed. USA: DAKO; 2012

29 Maleki Z, Shariat S, Mokri M, Atri M. ER-negative /PR-positive breast carcinomas or technical artifacts in immunohistochemistry? Arch Iran Med 2012;15(6):366-369

30 Krishnanand P, Kamath VV, Nagaraja A, Bandni M. Artifacts in oral mucosal biopsies. J Orofac Sci 2010;2(1):57-62

31 van der Velden U. Purpose and problems of periodontal disease classification. Periodontol 2000 2005;39:13-21

32 Mombelli A. Critical issues in periodontal diagnosis. Periodontol 2000 2005;39:9-12

33 Hujoel PP. Endpoints in periodontal trials: the need for an evidence-based research approach. Periodontol 2000 2004;36:196-204

34 Greenstein G. Clinical versus statistical significance as they relate to the efficacy of periodontal therapy. J Am Dent Assoc 2003;134(5):583-591

35 Addy M, Newcombe RG. Statistical versus clinical significance in periodontal research and practice. Periodontol 2000 2005;39:132-144

36 Barnett ML. Ethical issues in sponsored clinical research. J Dent Res 1995;74(4):1129-1132

37 Greco D, Diniz NM. Conflicts of interest in research involving human beings. J Int Bioethique 2008;19(1-2):143-154, 202-203

38 Aarons D. Research ethics. West Indian Med J 1995;44(4): $115-118$

39 Strech D. Evidence-based ethics-what it should be and what it shouldn't. BMC Med Ethics 2008;9:16

40 Kapp MB. Ethical and legal issues in research involving human subjects: do you want a piece of me? J Clin Pathol 2006;59(4):335-339

41 Bartold PM, Shi S, Gronthos S. Stem cells and periodontal regeneration. Periodontol 2000 2006;40:164-172

42 Ivanovski S, Gronthos S, Shi S, Bartold PM. Stem cells in the periodontal ligament. Oral Dis 2006;12(4):358-363

43 Human embryonic stem cell research. The Church of England. The ethical investment Advisory group April 2003 second edition

44 Karthikeyan BV, Pradeep AR. Gene therapy in periodontics: a review and future implications. J Contemp Dent Pract 2006;7(3):83-91

45 Mahale S, Dani N, Ansari SS, Kale T. Gene therapy and its implications in periodontics. Indian Soc Periodontol 2009;13:1-5

46 Abiko Y. Passive immunization against dental caries and periodontal disease: development of recombinant and human monoclonal antibodies. Crit Rev Oral Biol Med 2000;11(2): 140-158

47 Persson GR. Immune responses and vaccination against periodontal infections. J Clin Periodontol 2005;32(6, Suppl 6):39-53

48 Sharma DC, Prasad SB, Karthikeyan BV. Vaccination against periodontitis: the saga continues. Expert Rev Vaccines 2007;6(4): 579-590

49 Beevi L, Hedge S, Kashyap R, Kumar A. Vaccines and periodontal diseases-an insight. Dent Update 2009;36(10):635-638 